\title{
Exploring Airway Diseases by NMR-Based Metabonomics: A Review of Application to Exhaled Breath Condensate
}

\author{
Matteo Sofia, ${ }^{1}$ Mauro Maniscalco, ${ }^{1}$ Guglielmo de Laurentiis, ${ }^{1}$ Debora Paris, ${ }^{2}$ \\ Dominique Melck, ${ }^{2}$ and Andrea Motta ${ }^{2}$ \\ ${ }^{1}$ Department of Respiratory Medicine, A.O. Monaldi, University of Naples Federico II, 80131 Naples, Italy \\ ${ }^{2}$ Institute of Biomolecular Chemistry, National Research Council, 80078 Pozzuoli (Naples), Italy \\ Correspondence should be addressed to Mauro Maniscalco, mauromaniscalco@hotmail.com
}

Received 16 May 2010; Revised 27 December 2010; Accepted 16 January 2011

Academic Editor: Mika Ala-Korpela

Copyright ( $) 2011$ Matteo Sofia et al. This is an open access article distributed under the Creative Commons Attribution License, which permits unrestricted use, distribution, and reproduction in any medium, provided the original work is properly cited.

There is increasing evidence that biomarkers of exhaled gases or exhaled breath condensate (EBC) may help in detecting abnormalities in respiratory diseases mirroring increased, oxidative stress, airways inflammation and endothelial dysfunction. Beside the traditional techniques to investigate biomarker profiles, "omics" sciences have raised interest in the clinical field as potentially improving disease phenotyping. In particular, metabonomics appears to be an important tool to gain qualitative and quantitative information on low-molecular weight metabolites present in cells, tissues, and fluids. Here, we review the potential use of EBC as a suitable matrix for metabonomic studies using nuclear magnetic resonance (NMR) spectroscopy. By using this approach in airway diseases, it is now possible to separate specific EBC profiles, with implication in disease phenotyping and personalized therapy.

\section{Introduction}

Metabonomics is "the quantitative measurement of the dynamic multiparametric metabolic response of living systems to pathophysiological stimuli or genetic modification" [1] due to any exposure (including drug administration), lifestyle and environmental stress. It, therefore, appears to be a powerful tool to monitor possible changes in metabolic pathways, and measure the levels of biochemical molecules generated in a living system. Metabolites are small molecules with molecular mass $\leq 1 \mathrm{kD}[2]$ and are the end products of cellular activity. Observation of changes in metabolite concentrations may reveal the range of biochemical effects induced by a disease condition or its therapeutic intervention. The metabonomic analysis has two major potential applications, with implications in early diagnosis and disease phenotyping. It may also allow the recognition of unexpected or even unknown metabolites to formulate new pathophysiological hypotheses [3]. Moreover, the identification of individual metabolic characteristics could predict personal drug effectiveness and/or toxicity $[4,5]$.
The application of metabonomic analysis in chronic airway diseases has not been fully explored, but it holds a valid background. Several airway diseases, such as asthma or chronic obstructive pulmonary disease (COPD), which are largely spread in the population, cannot be qualified by a single biomarker and need a system biology analysis. Furthermore, other airway diseases such as cystic fibrosis (CF), although characterized by genetic abnormality, might be fruitfully investigated. Finally, the respiratory tract offers a natural matrix, the exhaled breath, which appears to be noteworthy for metabonomic analysis. Exhaled breath contains many different molecular species such as small inorganic molecules like nitric oxide (NO) or carbon monoxide (CO), volatile organic compounds (VOCs), and so forth, [6], which can be assayed in both the liquid and gaseous phases.

\section{NMR-Metabonomics}

The principal techniques used in metabonomics of breath ("breathomics") are mass spectrometry (MS) and nuclear magnetic resonance (NMR) spectroscopy, since they can 
handle complex biological samples with a high sensitivity, selectivity, and high throughput [7].

MS, usually combined with chromatographic separation methods, separates the molecules of a sample on the basis of their retention time and mass-to-charge ratio $\mathrm{m} / \mathrm{z}$, and their representation in a spectrum $[8,9]$.

Real-time measurements of breath are also possible using direct breathing ports and techniques such as proton transfer reaction-mass spectrometry (PTR-MS), selected ion flow tube-mass spectrometry (SIFT-MS), and ion mobility spectrometry (IMS), as well as other analytical techniques including chemical sensors and various forms of laser spectrometers [6]. MS metabonomics has recently been applied to CF, where airway inflammation brings about an increased production of reactive oxygen species, responsible for degradation of cell membranes and causing the formation of VOCs. Robroeks et al. [10] have evaluated if VOCs metabonomics, analyzed by gas chromatography-time of flight-mass spectrometry to assess VOC profiles, could discriminate $\mathrm{CF}$ and controls, and CF patients with and without Pseudomonas colonization. By using 22 VOCs, a $100 \%$ correct separation of CF patients and controls was possible, while with 10 VOCs, $92 \%$ of the subjects were correctly classified. The reproducibility of VOC measurements with a one-hour interval was very good. The authors concluded that metabonomics of VOCs in exhaled breath was possible in a reproducible way, and it was not only able to discriminate between CF patients and controls, but also between CF patients with or without Pseudomonas colonization.

NMR spectroscopy studies molecules by recording the interaction of a radiofrequency electromagnetic radiation with the nuclei (e.g., ${ }^{1} \mathrm{H},{ }^{13} \mathrm{C},{ }^{15} \mathrm{~N}$, etc.) placed in a strong magnetic field. A single nucleus in a molecule can be "observed" by monitoring the corresponding line (a "resonance") in an NMR spectrum, and the various parameters of that line (frequency, splitting, linewidth and amplitude) can be used to determine the molecular structure, conformation and dynamics of the molecule. In principle, assignment (i.e., identification) of NMR resonances for common metabolites could be possible by comparing the observed chemical shifts (i.e., the position of the line in a spectrum) with published reference data. When dealing with metabolites of unknown structure, chemical procedures for the separation of each molecule and use of two-dimensional NMR experiments (that spread signals in two dimensions) are required. Since NMR spectra show hundred of resonances, the presence of a discriminating element (e.g., a signal characteristic of a specific metabolite) in a series of spectra is often undetectable by visual inspection due to the inherent spectral complexity generated by line overlapping, and it is better highlighted by multivariate analysis (principal component analysis, PCA), which carefully identifies hidden phenomena and trends in ensembles of spectra [11]. The application of PCA to a group of spectra can immediately show whether all spectra behave similarly grouping in a single class, or fall apart into different groups. The main advantage of using NMR spectroscopy is its ability to provide a rapid and accurate metabolic picture of the sample with minimal sample pretreatment [12]. Furthermore, since the technique is nondestructive, the samples can be investigated several times as long as some preventative measures are taken to avoid metabolite degradation.

\section{Use of NMR Metabonomics for the Study of Airways}

Metabonomics has been employed to investigate several body fluids such as urine, plasma, serum, and tissue extracts as well as in-vivo cells and their extracts [13], but only few applications to airway diseases characterization have been reported.

Airway hyperreactivity (AHR), an important characteristic of airway pathophysiology in human asthma, has recently been evaluated in an animal model of asthma exacerbation by urine NMR-based metabonomics [14]. The authors assumed that airway dysfunction and inflammation would produce unique patterns of urine metabolites observed by high-resolution proton $\left({ }^{1} \mathrm{H}\right)$ NMR spectroscopy, and the data analyzed by multivariate statistical analysis. In this model, challenged (ovalbumin, administered intraperitoneally, plus ovalbumin aerosol) guinea pigs developed AHR and increased inflammation compared with sensitized or control animals. Partial least-squares discriminant analysis using concentration differences in metabolites could discriminate challenged animals with $90 \%$ accuracy. Noteworthy, urine metabonomic profiles were able to separate not only sensitized from challenged and from naïve animals, but also from animals treated with dexamethasone which improves AHR. Recently, Slupsky et al. demonstrated specific changes in NMR metabonomic urinary profiles during episodes of pneumonia caused by Streptococcus pneumoniae or Staphylococcus aureus [14].

NMR metabonomics was also used to study the mechanism behind the formation of airway biofilm caused by Pseudomonas aeruginosa, an infection particularly prevalent in patients with CF [15]. In this kind of patients, the sessile lifestyle, referred to as a biofilm, allows the antibiotic resistance and makes easier the process of colonization through the synthesis of sticky, polymeric compounds. In contrast, the planktonic, free-floating cells are more easily eradicated with antibiotics. In this study, chemical differences between planktonic and biofilm cells, based on ${ }^{1} \mathrm{H}-\mathrm{NMR}$, have been reported. In this study, NMR techniques have highlighted the metabolic differences between the two modes of growth in $P$. aeruginosa, and PCA, and spectral comparisons revealed that the overall metabolism of planktonic and biofilm cells displayed marked differences, which require more extensive NMR investigations.

More recently [16], metabolite profiles of bronchoalveolar lavage fluid (BALF) from pediatric patients with CF were correlated to the degree of airway inflammation using NMRbased metabonomics. BALF was collected from 11 children with CF during clinically indicated bronchoscopy. The BALF spectra with high levels of neutrophilic airway inflammation displayed signals from numerous metabolites whereas the spectra from subjects with low levels of inflammation were very sparse. The metabolites identified in samples taken from subjects with high inflammation include known markers of 
inflammation such as amino acids and lactate, as well as many novel signals. Statistical analysis highlighted the most important metabolites that distinguished the high- from the low-inflammation groups. This first demonstration of metabonomics of human BALF shows that clear distinctions in the metabolic profiles can be observed between subjects experiencing high versus low inflammation. However, the bronchoalveolar lavage has the important limitation of being invasive, requiring the introduction of exogenous fluid into alveolar space.

\section{EBC}

$\mathrm{EBC}$ is a noninvasive method of sampling the airways; it can be easily repeated and is acceptable to patients. Currently, EBC is used to measure biomarkers of airway inflammation and oxidative stress, and guidelines for its use have been recently published [17]. EBC can also be considered a matrix for analysis of environmental toxicants.

$\mathrm{EBC}$ collection requires the cooling of the exhaled breath (Figure 1(a)), resulting in a fluid sample that contains evaporated and condensed particles (water, ammonia, etc.) plus some droplets from the airway lining fluid $[17,18]$. These droplets are released by turbulent airflow and can be added to the water vapor from anywhere between the alveoli and the mouth. Therefore, not only volatiles, but also several other mediators with nonvolatile characteristics can be found in EBC samples, including adenosine, different interleukins $(-4,-5,-8)$, interferon- $\gamma$ [17]. EBC is mainly (>99\%) formed by water vapor, but also contains aerosol particles in which several other biomolecules including leukotrienes, 8isoprostane, prostaglandins, hydrogen peroxide, nitric oxidederived products, and hydrogen ions, can be detected [17]. EBC markers of oxidative stress such as hydrogen peroxide, isoprostanes, nitrogen oxides, $\mathrm{pH}$, ammonia, prostanoids and leukotrienes are increased in bronchial asthma [19]. EBC $\mathrm{pH}$ is lower in asthmatics and correlates well with sputum eosinophilia, total nitrate/nitrite, and oxidative stress [20], but did not reflect the clinical status of the patients. EBC markers that correlate with disease severity, response to treatment, or both are hydrogen peroxide, leukotrienes, 8 -isoprostane, nitrate, and nitrite [10]. It is assumed that airway surface liquid becomes aerosolized during turbulent airflow so that the content of the condensate reflects the composition of airway surface liquid, although large molecules may not aerosolize as well as small soluble molecules.

The major advantage of $\mathrm{EBC}$ is represented by the possibility to analyze both volatile and nonvolatile compounds [21]. There are some recent approaches to compare traditional blood test (glucose and urea) with the EBC in metabolic diseases. Accordingly, glucose in EBC from healthy volunteers was reproducible, unaffected by changes in salivary glucose, and increased during experimental hyperglycaemia [22].

Notably, EBC parameters are influenced by smoking, alcohol consumption, equipment, exercise, mode and rate of breathing, nasal contamination, environmental temperature and humidity, and assays used [23, 24], leading to undesirable variability. Exogenous contamination may also originate from the oral cavity. Ammonia and sulfur-containing compounds like $\mathrm{H}_{2} \mathrm{~S}$, methyl sulfide or mercaptans are released from the oral cavity, being produced by bacteria from different oral niches. However, oral sterilization before EBC collection or continuous saliva deglutition have been suggested to limit the effects of such contaminations [14]. The influence of age, sex, circadian rhythm, and infection remains unknown. Thus the analysis of EBC currently has important limitations.

Reference analytical techniques are required to provide definitive evidence for the presence of some inflammatory mediators in EBC and for their accurate quantitative assessment in this biological fluid. Finally, the physiological meaning and biochemical origin of most of volatile compounds are still not known, and biochemical pathways of their generation, origin, and distribution are only partly understood. Unfortunately, the concentrations of various mediators studied are very low, requiring highly sensitive assays.

\section{Metabonomics of EBC in Respiratory Diseases}

NMR-based metabonomics can be used to analyze EBC samples from adults, allowing a clear-cut separation between healthy subjects and patients with airway disease [11]. Although less sensitive than ELISA and MS, NMR spectroscopy requires minimal sample preparation with a rapid acquisition time of spectra $(10-15 \mathrm{~min})$. Furthermore, it shows a high degree of sensitivity $(\leq \mu \mathrm{mol} / \mathrm{L})$, and is nondestructive, allowing complete detection of metabolites present in the sample ("sample metabolic fingerprint") at a reasonable cost. NMR is also able to detect potential contamination of EBC from saliva, and examine the interfering effect of residual external contaminants, which is crucial for a correct $\mathrm{EBC}$ analysis of the variability of some biomarkers $[11,25,26]$.

To date there are several recommendations on the methodological approach to EBC collection, but its standardization is not completely defined, as EBC can be contaminated by metabolites originating from saliva as well as microbes present in the mouth $[27,28]$. We have recently proposed a possible protocol for EBC collection for NMR purposes (Figure 1) [11]. It requires that subjects breath through a mouthpiece and a two-way nonrebreathing valve, which also serve as a saliva trap, at normal frequency and tidal volume, while sitting comfortably and wearing a noseclip, for a period of 15 minutes (Figure 1(a)) [29]. They maintained a dry mouth during collection by periodically swallowing excess saliva. Condensate samples (1-2 mL) are immediately transferred into glass vials, closed with $20-\mathrm{mm}$ butyl rubber lined with PTFE septa, and crimped with perforated aluminum seals. Volatile substances, possibly deriving from extrapulmonary sources are removed by applying a gentle stream of nitrogen for 3 minutes before sealing $[30,31]$. Nitrogen was used because concentration of volatile solutes in EBC is dependent on their distribution between the saliva, exhaled air, and droplets, and the condensate, which can be altered by multiple factors including minute 


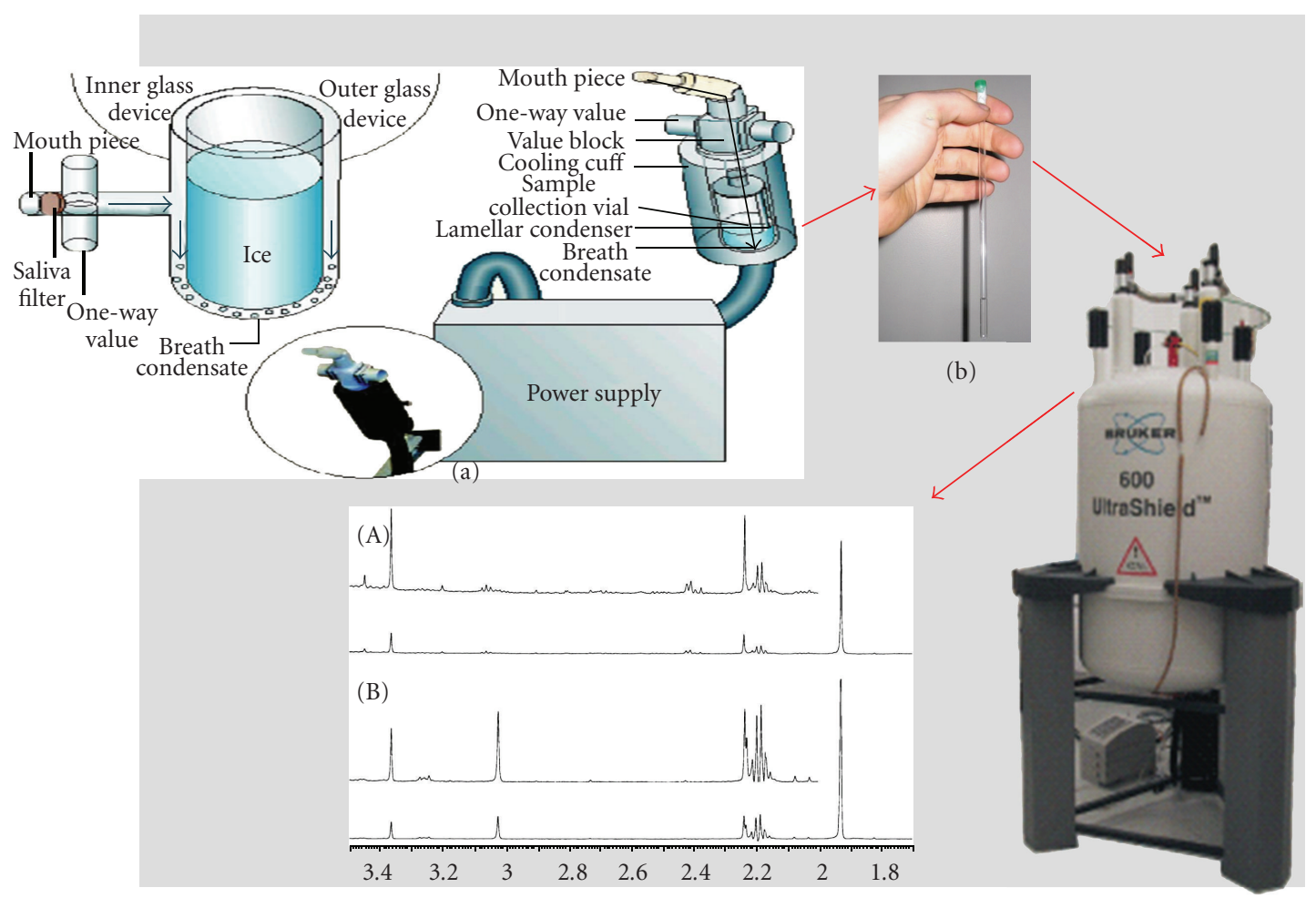

(d)

(c)

FIgURE 1: Metabonomics of EBC using NMR. The exhaled breath is cooled in (a), then transferred into the NMR tube (0.5-0.7 mL) (b) and put in the spectrometer $(\mathrm{c})$ to collect the spectra $(\mathrm{d})$.

ventilation, salivary $\mathrm{pH}$, solubility, temperature, and sample preparation [29]. Therefore, spectral differences may depend upon uncontrollable variables that prevent reliable quantification. The nitrogen stream also removes oxygen from solutions, which, together with freezing of sealed samples in liquid nitrogen, immediately "quenches" metabolism at the collection time, and prevents any metabolic decay [32, 33]. Samples are then stored at $-80^{\circ} \mathrm{C}$ until NMR analysis. Drying of the samples should be avoided to circumvent irreversible solute precipitation, and/or formation of insoluble aggregates, which we observed upon dissolving the dried condensate for NMR measurements.

Before NMR acquisition, EBC samples should be rapidly defrosted and transferred into the NMR tube (Figure 1(b)). To provide a field frequency lock for NMR acquisition, $70 \mu \mathrm{L}$ of a $\mathrm{D}_{2} \mathrm{O}$ solution [containing $1 \mathrm{mM}$ sodium 3-trimethylsilyl $\left[2,2,3,3-{ }^{2} \mathrm{H}_{4}\right]$ propionate (TSP) as a chemical shift and concentration reference for ${ }^{1} \mathrm{H}$ spectra, and sodium azide at $3 \mathrm{mM}$ ] are added to $630 \mu \mathrm{L}$ of condensate reaching $700 \mu \mathrm{L}$ of total volume.

Following acquisition (Figures $1(\mathrm{c})$ and $1(\mathrm{~d})$ ), ${ }^{1} \mathrm{H}-\mathrm{NMR}$ spectra are automatically data reduced to 200-250 integral segments ("buckets") using dedicated software packages (e.g., AMIX, Bruker Biospin, Germany). The resulting integrated regions are then used for statistical analysis and pattern recognition. To avoid possible errors in signal intensity due to difference in the volume of collected EBC samples, before pattern recognition analysis each integral region is normalized to the sum of all integral region of each spectrum. In the presence of contaminant peaks (e.g., those originating from the condenser disinfectant), which randomly alter the total area of the spectrum, each bucket has to be normalized to the TSP peak of known concentration, referring to a standard region comprised, for example, between 0.014 and -0.014 ppm.

Figure 2 represents spectra of saliva (left traces) and EBC samples (right traces) from a healthy subject (HS, lower spectra), a laryngectomized (middle spectra) and a COPD (top spectra) subjects. A visual examination establishes a striking correspondence between EBC spectra of HS and laryngectomized, suggesting that potential oral contamination (e.g., bacteria and/or saliva) is undetectable and, if present, beyond the sensitivity limit of NMR. By resorting to literature data [34] and two-dimensional NMR experiments, we identified all resonances present in EBC spectra (Figure 3). In saliva, signals between 3.3 and $6.0 \mathrm{ppm}$ originate from carbohydrates [35] and these are virtually absent in the EBC spectra (Figure 2). Compared to saliva, EBC spectra present fewer signals, but both saliva and EBC spectra appear to be different among the HS, laryngectomized and COPD classes considered (Figure 2); this is the basis for class separation in PCA based on NMR data.

A recent study [11] has evaluated the capability of NMR to separate EBC subjects with airway diseases (COPD) from subjects without respiratory diseases. Based on qualitative and quantitative spectral differences, five NMR signals 


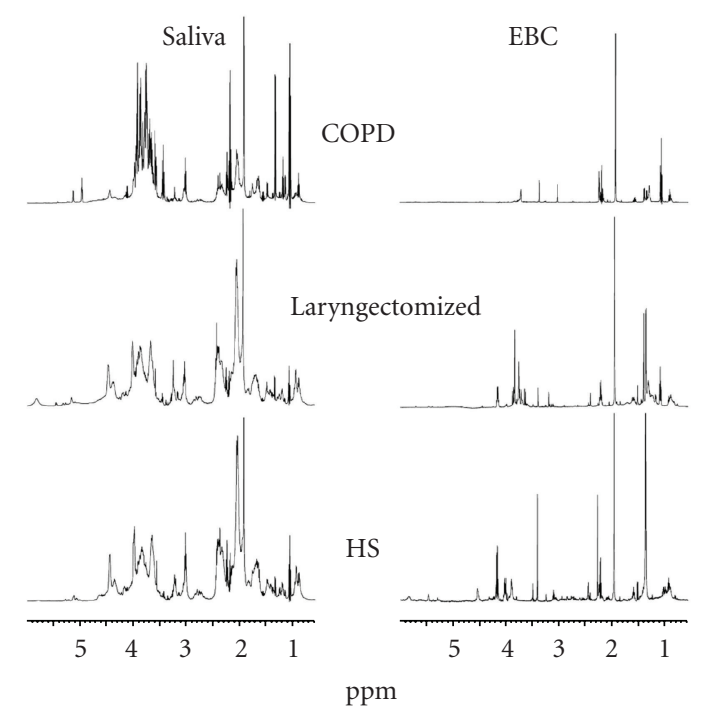

FIgURE 2: Representative one-dimensional ${ }^{1} \mathrm{H}-\mathrm{NMR}$ spectra from different patients. Spectra of saliva (left traces) and EBC samples (right traces) from a healthy (HS, lower traces), a laryngectomized (middle spectra), and a COPD (top spectra) subject are reported. Spectra were recorded on a Bruker Avance spectrometer operating at a frequency of $600.13 \mathrm{MHz}\left({ }^{1} \mathrm{H}\right)$ and equipped with a TCI CryoProbe. The water resonance was suppressed by using a specific pulse sequence designed to avoid intensity alteration of signals. The total acquisition time was $c a .10$ minutes per sample. Spectra were referred to TSP assumed to resonate at $\delta=0.00 \mathrm{ppm}$. In saliva spectra, the group of signals centered at $3.8 \mathrm{ppm}$ originates from carbohydrates, and is not visible in the corresponding EBC spectra.

appear to differentiate "respiratory" (COPD) from "nonrespiratory" (healthy and laryngectomized) subjects. It was also clearly proved that saliva and condensate have different profiles, with saliva contamination showing little influence on the interpretation of EBC by NMR-based metabonomics [11].

Likewise, Carraro et al. [36] reported the acetate signal variation as distinctive in asthmatic children with respect to controls, concluding that acetate increase might be related to increased acetylation of proinflammatory proteins in the extracellular space in the airway environment. Whether the metabonomic of exhaled breath condensate changes during systemic or metabolic disease is currently unknown.

NMR-based metabonomic analyses of EBC could clearly discriminate between asthmatic and healthy children, with 95\% success rate in their classification. Many authors believe that asthma should no longer be considered a single disease, and that efforts should be made to identify the different biochemical and inflammatory profiles behind asthma symptoms in order to treat them with specificallytargeted therapies [37]. Montuschi et al. [38] recently applied NMR-based metabonomics to discriminate between healthy individuals, patients with stable $\mathrm{CF}$, and cases of unstable $\mathrm{CF}$, showing that NMR is a powerful technique to monitor $\mathrm{EBC}$ in $\mathrm{CF}$.

In addition, we are currently applying NMR-based EBC metabonomics in other genetic airway diseases such as

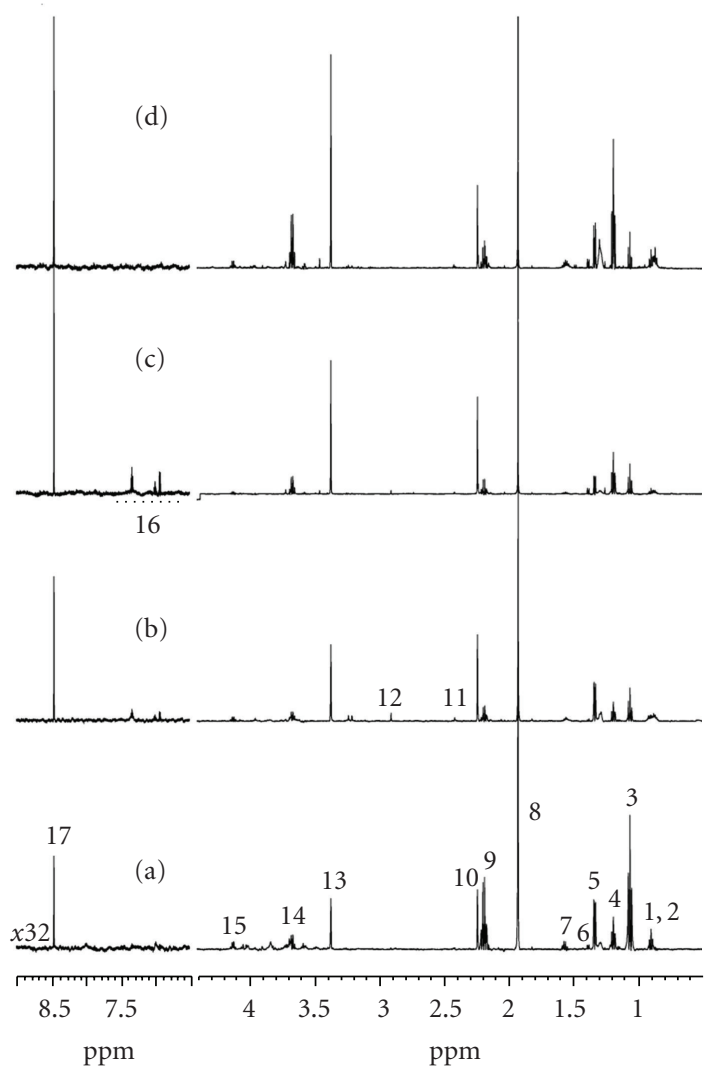

1, 2 Leu + n-Butyrate
3 Propionate
4 Thr
5 Lactate
6 Ala
7 n-Butyrate
8 Acetate
9 n-Butyrate

10 Pyruvate
11 Succinate
12 Trimethylamine
13 Methanol
14 Ethanol
15 Lactate
16 Phe
17 Formate

FIGURE 3: Resonance assignments of representative ${ }^{1} \mathrm{H}-\mathrm{NMR}$ spectra of EBC samples. The spectra of healthy (a), asthmatic (b), steroid-treated asthmatic (c), and COPD (d) patients are depicted. Peaks are labeled with progressive numbers, and assignments are listed below.

primary ciliary dyskinesia, in light of the diffusion of fast screening methods based on the exhaled NO on nasal or oral breath.

\section{Conclusions}

The power of NMR-based metabonomics has been shown for several biofluids, including blood, urine, and saliva. We believe that NMR metabonomics could also be applied to $\mathrm{EBC}$, which has the advantage of being noninvasive and reproducible; furthermore it shows a distinctive profile in comparison to saliva, thus supporting its origin from lower airways. Moreover, EBC metabonomic analysis is applied to a living matrix in the absence of external induced perturbations that may represent important preanalytical variable with conventional assay measuring single compound. 
There is only limited experience with metabonomics on EBC in humans, but reproducibility of method has been successfully assessed, and useful protocols to differentiate metabolic profile of patients with asthma, COPD, or cystic fibrosis have been reported. However, more studies are needed to show, if true, that the holistic approach of EBC metabonomics may be a progress over the traditional reductionistic approach in chronic airway disease.

\section{References}

[1] J. K. Nicholson, J. C. Lindon, and E. Holmes, "“Metabonomics": understanding the metabolic responses of living systems to pathophysiological stimuli via multivariate statistical analysis of biological NMR spectroscopic data," Xenobiotica, vol. 29, no. 11, pp. 1181-1189, 1999.

[2] M. E. Bollard, E. G. Stanley, J. C. Lindon, J. K. Nicholson, and E. Holmes, "NMR-based metabonomic approaches for evaluating physiological influences on biofluid composition," NMR in Biomedicine, vol. 18, no. 3, pp. 143-162, 2005.

[3] U. Sauer, M. Heinemann, and N. Zamboni, "Getting closer to the whole picture," Science, vol. 316, no. 5824, pp. 550-551, 2007.

[4] J. M. Meyer and G. S. Ginsburg, "The path to personalized medicine," Current Opinion in Chemical Biology, vol. 6, no. 4, pp. 434-438, 2002.

[5] T. A. Clayton, J. C. Lindon, O. Cloarec et al., "Pharmacometabonomic phenotyping and personalized drug treatment," Nature, vol. 440, no. 7087, pp. 1073-1077, 2006.

[6] W. M. Amann, J. Pleil, T. Risby, and J. Schubert, "Methodological issues of sample collection and analysis of exhaled breath," European Respiratory Monthly, vol. 49, pp. 96-114, 2010.

[7] M. Coen, E. Holmes, J. C. Lindon, and J. K. Nicholson, "NMRbased metabolic profiling and metabonomic approaches to problems in molecular toxicology," Chemical Research in Toxicology, vol. 21, no. 1, pp. 9-27, 2008.

[8] K. Dettmer, P. A. Aronov, and B. D. Hammock, "Mass spectrometry-based metabolomics," Mass Spectrometry Reviews, vol. 26, no. 1, pp. 51-78, 2007.

[9] X. Feng, X. Liu, Q. Luo, and B. I. F. Liu, "Mass spectrometry in systems biology: an overview," Mass Spectrometry Reviews, vol. 27, no. 6, pp. 635-660, 2008.

[10] C. M. H. H. T. Robroeks, J. J. B. N. Van Berkel, J. W. Dallinga et al., "Metabolomics of volatile organic compounds in cystic fibrosis patients and controls," Pediatric Research, vol. 68, no. 1, pp. 75-80, 2010.

[11] G. de Laurentiis, D. Paris, D. Melck et al., "Metabonomic analysis of exhaled breath condensate in adults by nuclear magnetic resonance spectroscopy," European Respiratory Journal, vol. 32, no. 5, pp. 1175-1183, 2008.

[12] E. J. Saude, I. P. Obiefuna, R. L. Somorjai et al., "Metabolomic biomarkers in a model of asthma exacerbation: urine nuclear magnetic resonance," American Journal of Respiratory and Critical Care Medicine, vol. 179, no. 1, pp. 25-34, 2009.

[13] O. Beckonert, H. C. Keun, T. M. Ebbels et al., "Metabolic profiling, metabolomic and metabonomic procedures for NMR spectroscopy of urine, plasma, serum and tissue extracts," Nature protocols, vol. 2, no. 11, pp. 2692-2703, 2007.

[14] C. M. Slupsky, A. Cheypesh, D. V. Chao et al., "Streptococcus pneumoniae and Staphylococcus aureus pneumonia induce distinct metabolic responses," Journal of Proteome Research, vol. 8, no. 6, pp. 3029-3036, 2009.
[15] E. L. Gjersing, J. L. Herberg, J. Horn, C. M. Schaldach, and R. S. Maxwell, "NMR metabolomics of planktonic and biofilm modes of growth in Pseudomonas aeruginosa," Analytical Chemistry, vol. 79, no. 21, pp. 8037-8045, 2007.

[16] J. E. Wolak, C. R. Esther, and T. M. O’Connell, "Metabolomic analysis of bronchoalveolar lavage fluid from cystic fibrosis patients," Biomarkers, vol. 14, no. 1, pp. 55-60, 2009.

[17] I. Horváth, J. Hunt, P. J. Barnes et al., "Exhaled breath condensate: methodological recommendations and unresolved questions," European Respiratory Journal, vol. 26, no. 3, pp. 523-548, 2005.

[18] S. A. Kharitonov and P. J. Barnes, "Exhaled markers of pulmonary disease," American Journal of Respiratory and Critical Care Medicine, vol. 163, no. 7, pp. 1693-1722, 2001.

[19] R. Accordino, A. Visentin, A. Bordin et al., "Long-term repeatability of exhaled breath condensate $\mathrm{pH}$ in asthma," Respiratory Medicine, vol. 102, no. 3, pp. 377-381, 2008.

[20] K. Ganas, S. Loukides, G. Papatheodorou, P. Panagou, and N. Kalogeropoulos, "Total nitrite/nitrate in expired breath condensate of patients with asthma," Respiratory Medicine, vol. 95, no. 8, pp. 649-654, 2001.

[21] M. Maniscalco, G. De Laurentiis, C. Pentella et al., "Exhaled breath condensate as matrix for toluene detection: a preliminary study," Biomarkers, vol. 11, no. 3, pp. 233-240, 2006.

[22] E. H. Baker, N. Clark, A. L. Brennan et al., "Hyperglycemia and cystic fibrosis alter respiratory fluid glucose concentrations estimated by breath condensate analysis," Journal of Applied Physiology, vol. 102, no. 5, pp. 1969-1975, 2007.

[23] K. Czebe, I. Barta, B. Antus, M. Valyon, I. Horváth, and T. Kullmann, "Influence of condensing equipment and temperature on exhaled breath condensate $\mathrm{pH}$, total protein and leukotriene concentrations," Respiratory Medicine, vol. 102, no. 5, pp. 720-725, 2008.

[24] T. Kullmann, I. Barta, B. Antus, M. Valyon, and I. Horváth, "Environmental temperature and relative humidity influence exhaled breath condensate $\mathrm{pH}$," European Respiratory Journal, vol. 31, no. 2, pp. 474-475, 2008.

[25] P. Montuschi, S. A. Kharitonov, G. Ciabattoni, and P. J. Barnes, "Exhaled leukotrienes and prostaglandins in COPD," Thorax, vol. 58, no. 7, pp. 585-588, 2003.

[26] P. Montuschi, "Exhaled breath condensate analysis in patients with COPD," Clinica Chimica Acta, vol. 356, no. 1-2, pp. 2234, 2005.

[27] P. Latzin, J. Beck, A. Bartenstein, and M. Griese, "Comparison of exhaled breath condensate from nasal and oral collection," European Journal of Medical Research, vol. 8, pp. 505-510, 2003.

[28] J. Chladkova, I. Krcmova, J. Chladek, P. Cap, S. Micuda, and Y. Hanzalkova, "Validation of nitrite and nitrate measurements in exhaled breath condensate," Respiration, vol. 73, no. 2, pp. 173-179, 2006.

[29] R. M. Effros, "Exhaled breath condensate pH," American Journal of Respiratory and Critical Care Medicine, vol. 173, no. 9, pp. 1047-1048, 2006.

[30] R. M. Effros, K. W. Hoagland, M. Bosbous et al., "Dilution of respiratory solutes in exhaled condensates," American Journal of Respiratory and Critical Care Medicine, vol. 165, no. 5, pp. 663-669, 2002.

[31] C. L. Whittle, S. Fakharzadeh, J. Eades, and G. Preti, "Human breath odors and their use in diagnosis," Annals of the New York Academy of Sciences, vol. 1098, pp. 252-266, 2007.

[32] J. D. Bell, J. C. Brown, and P. J. Sadler, "NMR studies of body fluids," NMR in Biomedicine, vol. 2, no. 5-6, pp. 246-256, 1989. 
[33] O. Beckonert, H. C. Keun, T. M. Ebbels et al., "Metabolic profiling, metabolomic and metabonomic procedures for NMR spectroscopy of urine, plasma, serum and tissue extracts," Nature protocols, vol. 2, no. 11, pp. 2692-2703, 2007.

[34] T. W. M. Fan, "Metabolite profiling by one- and twodimensional NMR analysis of complex mixtures," Progress in Nuclear Magnetic Resonance Spectroscopy, vol. 28, no. 2, pp. 161-219, 1996.

[35] M. Grootveld and C. J. L. Silwood, "1H-NMR analysis as a diagnostic probe for human saliva," Biochemical and Biophysical Research Communications, vol. 329, no. 1, pp. 15, 2005.

[36] S. Carraro, S. Rezzi, F. Reniero et al., "Metabolomics applied to exhaled breath condensate in childhood asthma," American Journal of Respiratory and Critical Care Medicine, vol. 175, no. 10, pp. 986-990, 2007.

[37] C. Auffray, I. M. Adcock, K. F. Chung, R. Djukanovic, C. Pison, and P. J. Sterk, "An integrative systems biology approach to understanding pulmonary diseases," Chest, vol. 137, no. 6, pp. 1410-1416, 2010.

[38] P. Montuschi, D. Paris, D. Melck, V. Lucidi, and A. Motta, "Metabolomic analysis by nuclear magnetic resonance spectroscopy of exhaled breath condensate in patient with cystic fibrosis," European Respiratory Journal, vol. 34, supplement 53, p. 63s, 2009. 

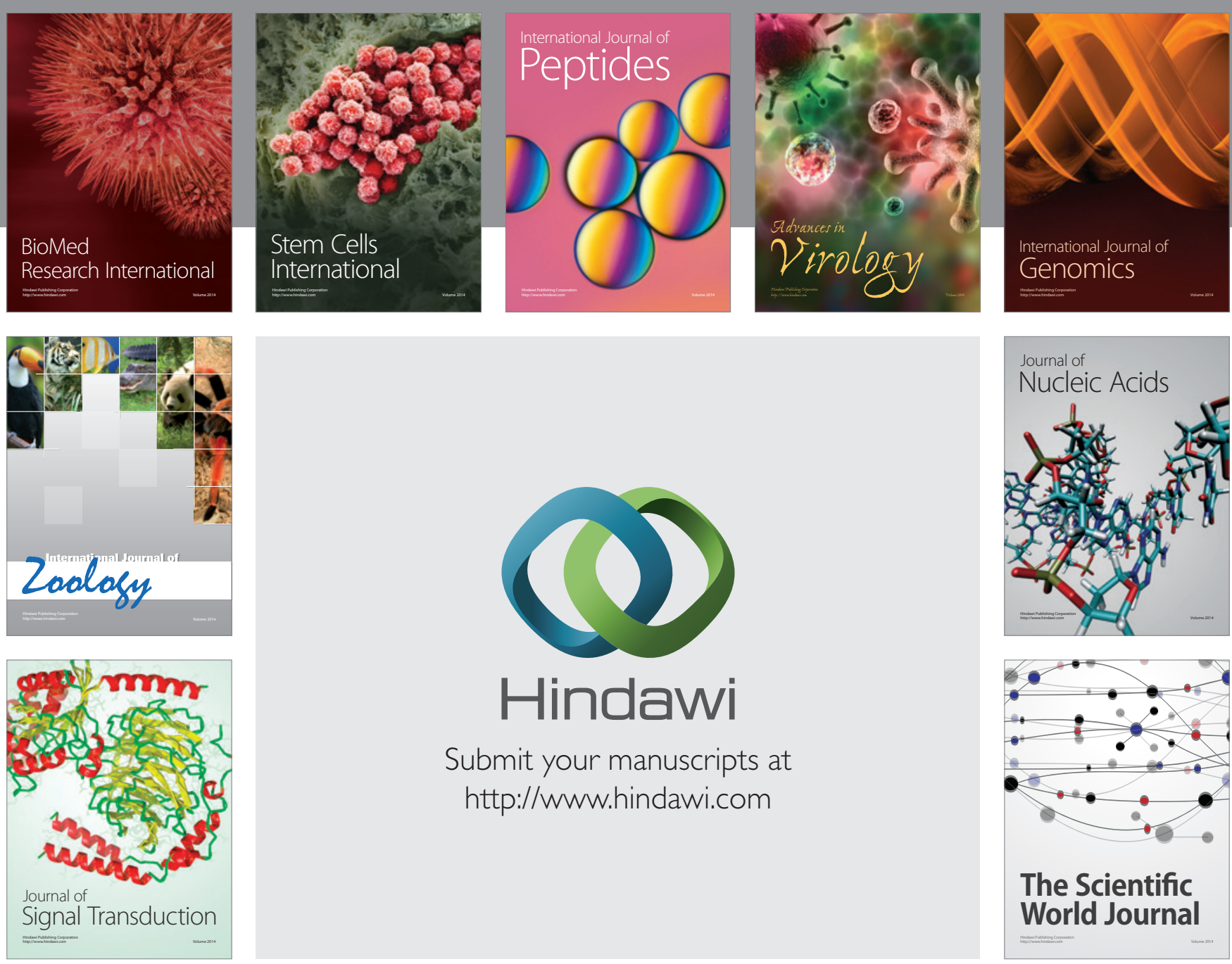

Submit your manuscripts at

http://www.hindawi.com
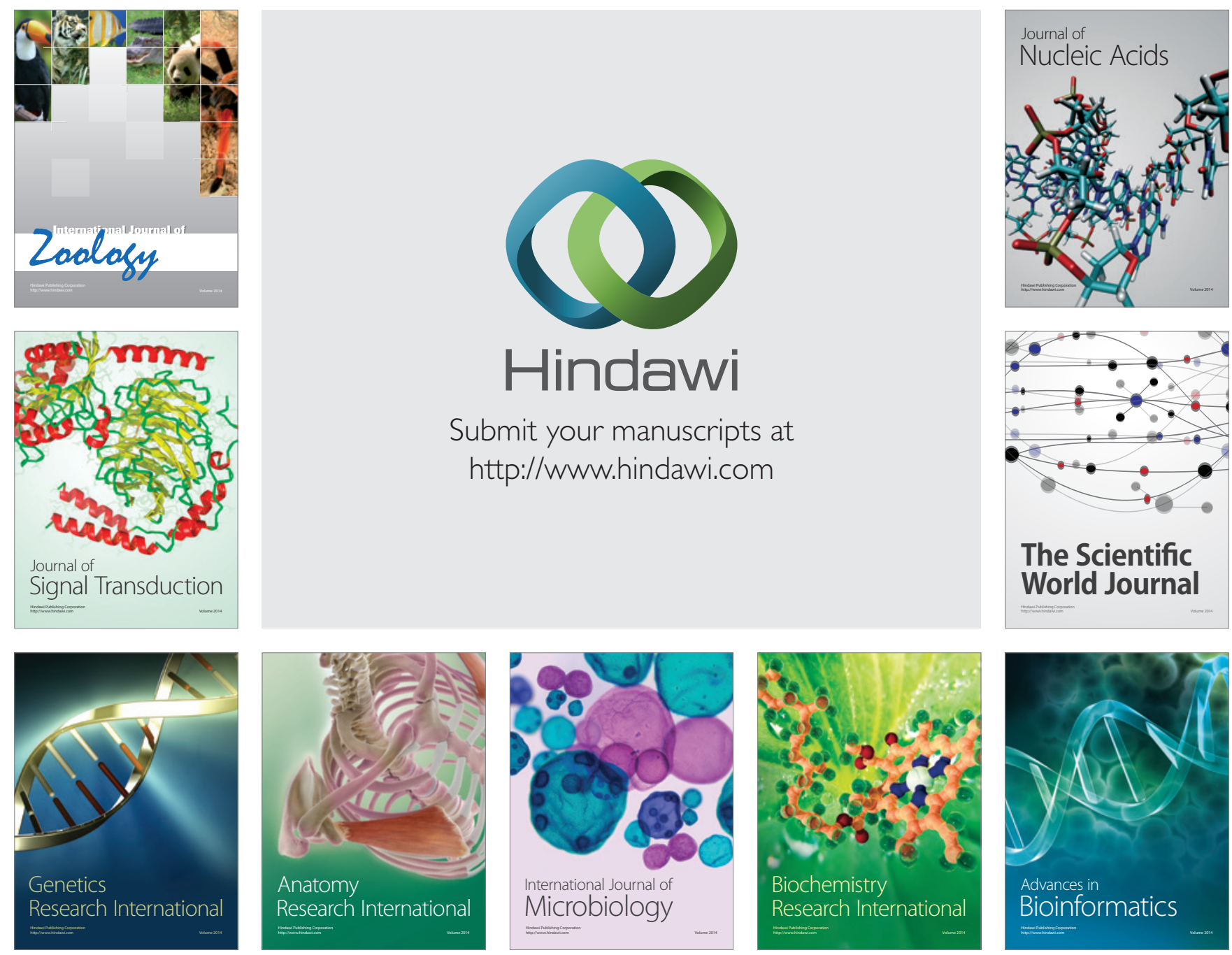

The Scientific World Journal
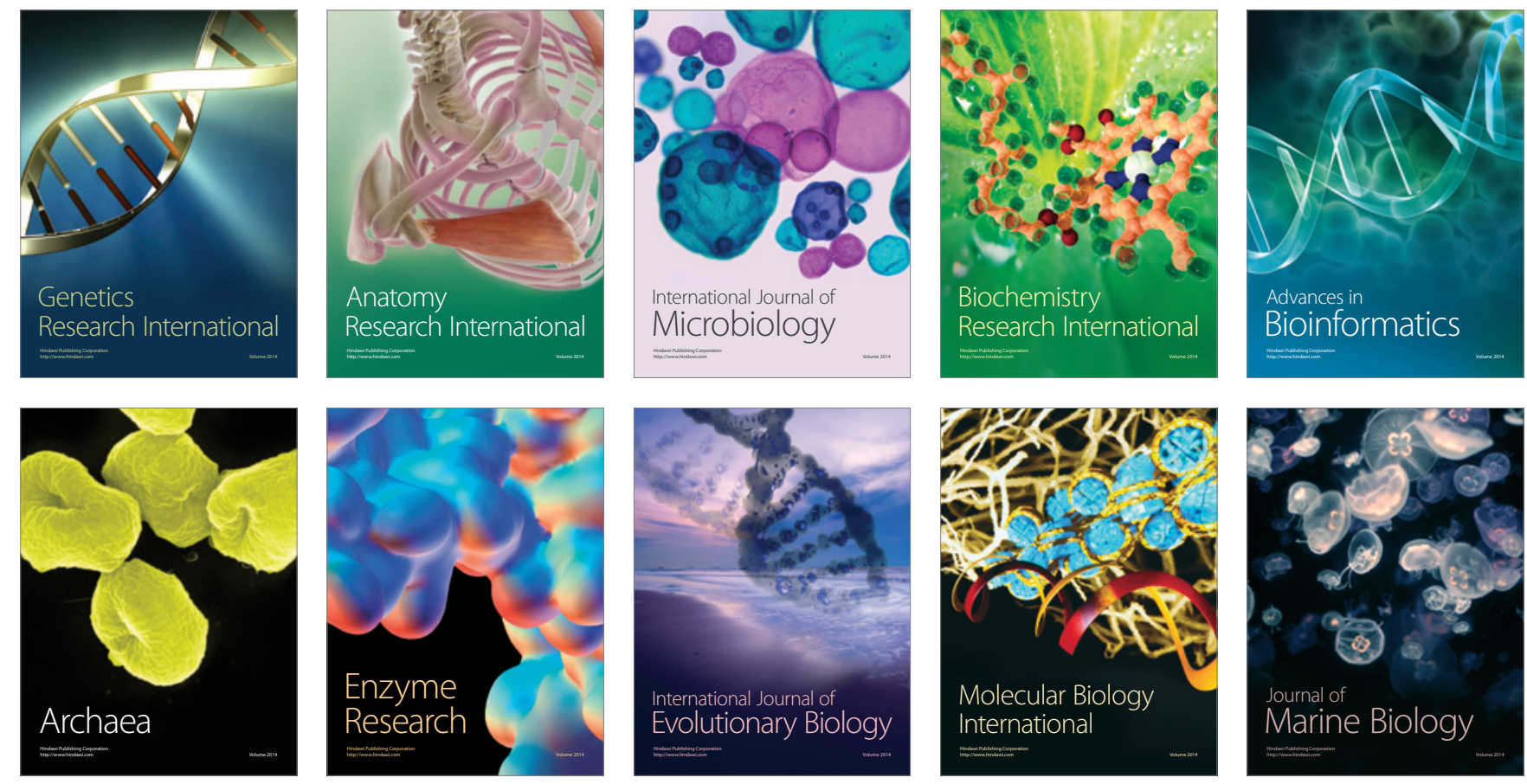